\title{
Design of Safety FPGA Optimization Internet of things and Its Reliability Analysis
}

\author{
Lan LUAN ${ }^{1, a}$ \\ ${ }^{1}$ Department of Computer Science College of Computer Science and Technology Guizhou \\ University, Guizhouguiyang, 550002, China
}

Keywords: IOT; Welding Machine Monitoring System; Burst Detection; Sliding Window

\begin{abstract}
Aiming at demands based on abnormal condition detection and warning in welding machine monitoring system of IOT, put forward a burst detection algorithm for multi-population firefly, achieve optimization selection and configuration of sliding window size and improve the processing speed and detection performance of burst detection model through collaborative work of different species of fireflies. Simulation results show that burst detection algorithm based on multi-population firefly has less processing time and higher accuracy and recall rate than the traditional burst detection algorithm under the condition of same burst probability or maximum sliding window size.
\end{abstract}

\section{Introduction}

In order to preferably realize the fine management of the welding site and the level of lean manufacturing, it is necessary to apply information technology to improve the welding efficiency and reduce the energy consumption in the whole process of welding. Welding machine monitoring system based on Internet of things can monitor machine working state and related information in real time, realize the calculation and fine management of basic data such as welding hours, volume and energy consumption, supervise the welding condition of the operator and promptly manage non-normative welding of the operator, and can analyze the reasons for the failure of the welding machine through historical alarm information on abnormal state of welding machine, while abnormal data in welding machine monitoring system can be identified correctly by burst detection through data stream of IOT. This paper will put forward a burst detection algorithm based on multi-population firefly to realize the optimization of abnormal data detection in welding machine monitoring system based on IOT through collaborative work of different species of fireflies, so as to reduce the false positive rate and false negative rate of burst detection under the abnormal condition of welding data stream and improve the early warning effect.

\section{Burst Detection Model and Algorithm}

\section{Definition of burst detection model and problem}

Fig. 1 depicts the burst detection model of multi source data streams in the welding machine monitoring system based on IOT. After the data stream arrives at the network node, first, features are extracted and classified with classifier, and then are placed into corresponding burst detection queue by the scheduler, and after processing of multithreaded operation processor, the results will be sent to the route selector and the data direction will be decided: deliver to storage manager for filing or output. Load equalizer continuously detects the output performance; if the performance is too low, take measures to reduce the system load until it reaches the standard. As the rate of data stream arriving at network node is dynamic and changed, even though the average load of a node is not very high, a node will be likely to encounter temporary load peak and its waiting time for data processing will be affected in the peak period. Therefore, in order to minimize the data processing time, the load balancing strategy is needed to avoid temporary overload. 


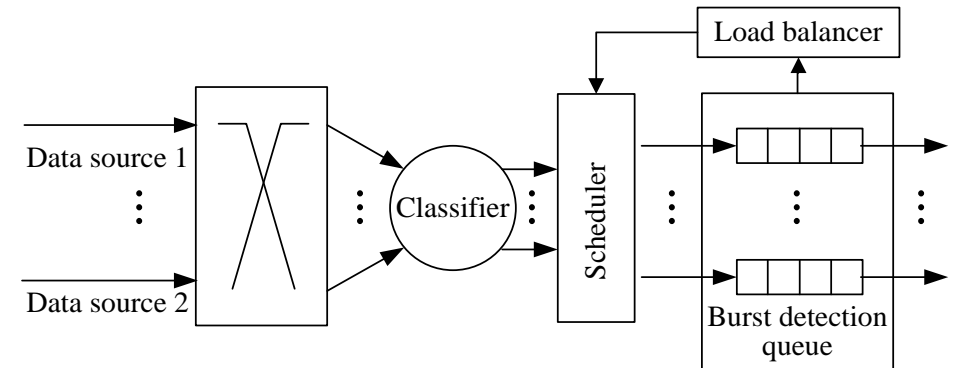

Fig.1. Burst detection model of multi-source heterogeneous data stream

Definition 1: given set $E=\{e 1, e 2, \ldots, e n\}$ of $n$ events on time sequence $t=0,1, \ldots, T$ of $T$ units, data stream comes from $x$ sensor nodes and event sequence on node $j$ is represented as $E j=\{e 1 j, e 2 j, \ldots, e n j\}$, then event set $E$ is represented as:

$E=\sum_{j=1}^{x} E_{j}$

Assumed that event arrival rate is $\lambda$ and each event is independent and identically distributed, the number of events at any time is subject to the Poisson distribution:

$P(n \mid \lambda)=e^{-\lambda} \frac{\lambda^{n}}{n !}, n=0,1,2, \ldots$

Assumed that events arrived at different time are independent, the probability distribution of the number $\{\mathrm{N} 1, \mathrm{n} 2, \ldots$ For $\mathrm{nT}\}$ of events in the event flow is:

$$
P\left(n_{1}, n_{2}, \ldots, n_{T} \mid \lambda_{1}, \lambda_{2}, \ldots, \lambda_{T}\right)=\prod_{t=1}^{T} P\left(n_{t} \mid \lambda_{t}\right)
$$

Definition 2: when the number $B$ of events in the event set $E$ on time sequence $T$ is greater than or less than one order of magnitude of average event count, it is known as burst, at this moment, $B>\mu E+c \sigma E$ or $B<\mu E-c \sigma E$, where $\mu$ is the mean value, $c$ is constant and $\sigma$ is the standard deviation.

The distribution of Gauss density function in D-dimensional space is defined as:

$$
p\left(x, M_{j}, C_{j}\right)=\frac{1}{\sqrt{\left((2 \pi)^{d}|C|\right)}} e^{-\left(x-M_{j}\right)^{T} C_{j}\left(x-M_{j}\right)}
$$

Where, $M_{j}$ represents $d$-dimensional average vector and $C_{j}$ is a $d \times d$ diagonal matrix representing covariance vector.

Under the condition of single information source, maximize the utility function $U(x, a)$ (where, $a$ $\in S_{e}, S_{e}$ is a set for all possible execution), and when the utility function represents a measurement of the utility brought from performing $a$ when the true state is a particular value $x$. The expected utility is expressed by $\beta$ :

$$
\beta\left(p\left(x \mid Z^{k}\right), a\right)=\int\{U(x, a)\} p\left(x \mid Z^{k}\right) d x
$$

The optimal execution $\bar{a}$ is a strategy that maximizes the expected utility, namely:

$$
\bar{a}=\max \beta\left(p\left(x \mid Z^{k}\right), a\right)
$$

Formula (6) is equivalent to the maximum of the following formula:

$$
\int U(x, a) p\left(Z^{k} \mid x\right) p(x) d x
$$

When regular report time arrives, abnormal situations are detected or data query request is received in the current formula system, the information obtained from sensing unit is converted and preliminarily processed through $\mathrm{A} / \mathrm{D}$ to form monitoring data packet at the sensor nodes in line with the conditions; monitoring data packet is transmitted to convergent node through one-hop or multi-hop wireless and then transmitted and connected to IP backbone network through one-hop or multi-hop wireless, and finally transmitted to post-formula system through IP backbone network; data processing model unit of post-formula system processes monitoring data received in accordance with the burst detection algorithm to determine whether the welding condition of welding machine is abnormal, so that early warning is made promptly.

\section{Burst detection algorithm}

In the burst detection algorithm based on multi-population firefly proposed in this paper, fireflies 
of each population are responsible for monitoring an attribute of physical quantity, and two elements of the basic firefly algorithm are contained: fluorescein brightness and attraction. Fluorescein brightness is related to fitness value of objective function at the location of firefly, while the fitness value of firefly at some point is the function of sliding window size adopted when burst detection is carried out at the node where the firefly is. The degree of attraction determines the distance of firefly with weak brightness moving to firefly with strong brightness, namely, the amplitude of change of sliding window size at the node where firefly with weak brightness is. Through constant updating of fluorescein brightness and attraction of each firefly, realize the optimization of the sliding window size for burst detection, so as to improve the processing speed and detection performance of burst detection model. The optimization mechanism of firefly algorithm is described as follows from the view of mathematics:

Definition 3: fluorescein brightness of firefly is defined as:

$$
I(r)=I_{0} e^{-\gamma \cdot r^{2}}
$$

Where, $I_{0}$ is fluorescein brightness of firefly itself ( $\mathrm{r}=0$ ), which is related to objective function value $\varphi$, and the greater the value $\varphi$ is, the higher the fluorescein brightness of itself is; $\gamma$ is fluorescein absorption coefficient, which is used to represent the gradual decay rate of fluorescein brightness with the increase of the distance and can be set as a constant. $r$ represents the distance between fireflies.

Definition 4: attraction of firefly is defined as:

$$
\beta(r)=\beta_{0} e^{-\gamma \cdot r^{2}}
$$

Where, $\beta_{0}$ is attraction when $r=0, \gamma$ and $r$ have the same meaning as above. When $r=1 / \sqrt{\gamma}$, it is known as the characteristic distance, at this moment, the attraction will be significantly changed from $\beta_{0}$ to $\beta_{0} e^{-1}$.

Assumed that the sliding window size is respectively $x_{i}$ and $x_{j}$ at the node where any two fireflies I and $\mathrm{J}$ are, the distance between two fireflies is expressed as $r_{i j}=\left\|x_{i}-x_{j}\right\|$, and displacement from firefly $i$ being attracted to the direction of firefly $j$ with higher fluorescein brightness is defined as:

$$
\Delta x_{i}=\beta_{0} e^{-r^{2}}\left(x_{j}^{t}-x_{i}^{t}\right)+\alpha \cdot \varepsilon_{i}, \quad x_{i}^{t+1}=x_{i}^{t}+\Delta x_{i}
$$

Where, $\alpha$ is the step size factor, which is a random parameter. $\varepsilon_{i}$ is a random number vector subject to Gauss distribution.

\section{Simulation Results and Analysis}

The simulation environment of this paper uses MICA $2^{[13]}$ node produced by Crossbow Company as the standard, and the communication distance of the node is 50 meters. In the process of simulation, random data is used to simulate the sensing data of wireless sensor network node, including welding current and electrode voltage. The specific methods are as follows: firstly, the value $v$ of each node in each dimension is initialized randomly as a value in the normal range. In each round of the following experiments, the maximum variation amplitude of value $v$ is $f(f>0)$ compared with previous round. Supposed that the value on $i^{\text {th }}$ dimension in $r^{\text {th }}$ round is $v(r)$ [i], value change in $r+1^{\text {th }}$ round is $v(r)[i]+\operatorname{rand}(-f, f)$. Other experimental parameters and default values are shown in Table 1.

Tab.1. Experimental parameters and default values

\begin{tabular}{ll}
\hline Experimental parameter & Default value \\
\hline Number of sensor node & 80 \\
Average degree of network node & 5 \\
Number of convergent node & 4 \\
Packet size & 40 bytes \\
Welding current range & $3.3-6.0 \mathrm{kA}$ \\
Electrode voltage range & $2.1-5.1 \mathrm{kN}$ \\
Welding data set size & 86281 \\
\hline
\end{tabular}

Table 1 shows the parameters and default values of the simulation experiment. This paper will 
compare the performance on indicators including processing time, Precision and recall by SBT, SAT and MPF based on multi-populations firefly. The accuracy rate refers to the ratio of the number of real detected bursts to the number of all real bursts; the recall ratio refers to the ratio of the number of real detected bursts to the number of detected bursts.

The first group of experiments compares the processing time of different burst detection algorithms under different burst probability. In this group of experiments, the maximum sliding window size is 64 and the simulation results are shown in Fig. 2. With the increase of burst probability, the processing time of 3 kinds of burst detection algorithms will increase. In the case of same burst probability, the average processing time of MPF is 16.8\% less than that of SAT and $42.7 \%$ less than that of SBT.

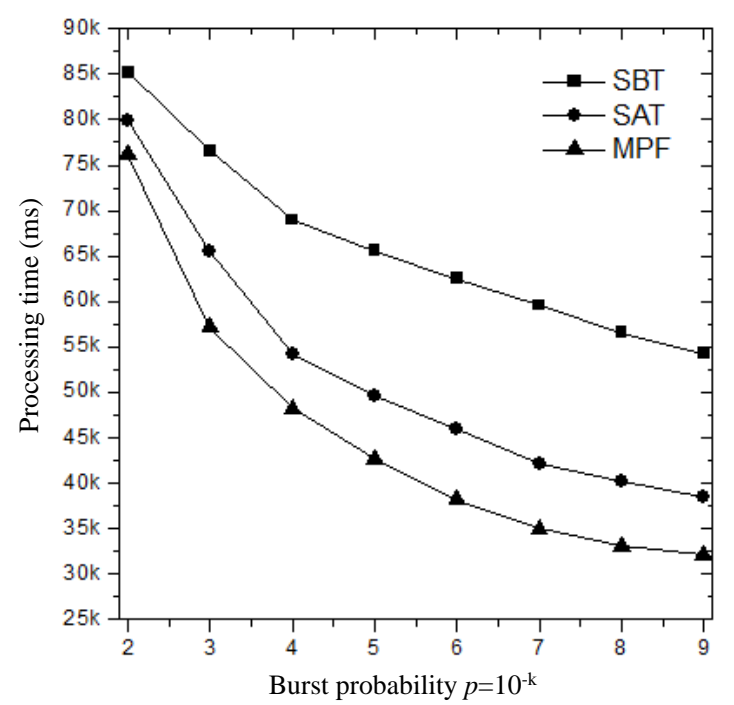

Fig.2. Processing time of burst detection under different burst probability

The second group of experiments compares the relationship between the processing time of different burst detection algorithms and the maximum sliding window size. The simulation results are shown in Fig. 3. The processing time of 3 kinds of burst detection algorithms is directly proportional to the maximum sliding window size. In the case of same sliding window size, processing time of MPF is less than that of SAT and SBT and with the increase of sliding window size, the performance advantage of MPF is more obvious. When the maximum size of the sliding window is 8 , the processing time of MPF is equivalent to $61.1 \%$ of processing time of SAT and $50.6 \%$ of processing time of SBT; when the maximum slide window size is 512 , the processing time of MPF is only equivalent to $39.5 \%$ of processing time of SAT and $54.3 \%$ of processing time SBT.

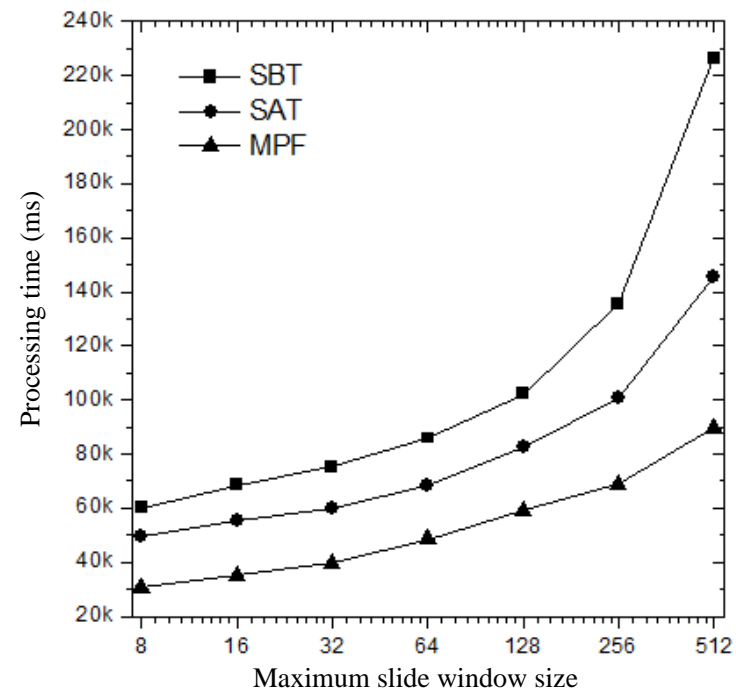

Fig.3. Effect of maximum sliding window size on processing time 
The third group of experiments mainly compares the effect of the maximum sliding window size on the accuracy and the simulation results are shown in Fig. 4. With the increase of the maximum sliding window size, the accuracy of 3 kinds of burst detection algorithms has increased. However, when the maximum sliding window size is more than 128, the rising amplitude in accuracy of 3 kinds of burst detection algorithms slows down significantly. The accuracy of MPF is higher than that of SAT and SBT in the case of same maximum sliding window size.

\section{Conclusion}

As a common characteristic in burst detection algorithm of data stream, the speed and accuracy as the two most key parameters of data stream algorithm are always some kind of paradox, for which a good compromise needs to be got when designing the algorithm. In general, the processing time of burst detection algorithm is directly proportional to the maximum sliding window size. With the increase of the maximum sliding window size, the processing time will be longer and the burst detection speed will be slower, but the accuracy will increase to some extent at the same time. Different from the change rule of the accuracy, the recall rate presents a trend of increasing first and then decreasing in the process of increasing the maximum sliding window size, which is related to the slow rising amplitude of accuracy in the later stage. Further research work will take the false positive and false negative problems of burst detection algorithm into account to minimize the combination of false positive rate / false negative rate through the optimization of existing algorithms.

\section{Acknowledgement}

The youth fund project of Guizhou university in 2010 "The AES Algorithm Realization based on FPGA” 2010038

\section{References}

[1] Jinyu Hu, Zhiwei Gao and Weisen Pan. Multiangle Social Network Recommendation Algorithms and Similarity Network Evaluation[J]. Journal of Applied Mathematics, 2013 (2013).

[2] Jinyu $\mathrm{Hu}$ and Zhiwei Gao. Modules identification in gene positive networks of hepatocellular carcinoma using Pearson agglomerative method and Pearson cohesion coupling modularity[J]. Journal of Applied Mathematics, 2012 .

[3] Geng, Y., Chen, J., Fu, R., Bao, G., and Pahlavan, K. Enlighten Wearable Physiological Monitoring systems: On-Body RF Characteristics Based Human Motion Classification Using a Support Vector Machine. PP(99), 1-16.

[4] Song, X., and Geng, Y.Distributed Community Detection Optimization Algorithm for Complex Networks. Journal of Networks, 9(10), 2758-2765. 